\title{
A review on methods used to record and analyze microfluctuations of the accommodation in the human eye
}

\author{
Pier Paolo Monticone \\ pmonticone@ethz.ch
}

\section{Marino Menozzi}

In steady state conditions, the accommodation of the human eye presents fluctuations. These fluctuations, called microfluctuations, are described in the literature as having an amplitude of less than $1 \mathrm{D}$ and a frequency up to a few Hz. Since Collins' report about microfluctuations in 1937 [1], several methods have been used to record and analyze microfluctuations. Results reported in the literature are partially in disagreement; we show how these disagreements could be due to the different methodologies used to record and analyze the accommodation signals. We classify and discuss properties of instruments used to record the microfluctuations and methods used to elaborate the resulting signals. In particular, we catalog the measuring instruments in three families, i.e. wave-front aberrometer, classical IR autorefractometer, and ultrasound; while other three families are defined for the methods of analysis: root mean square (RMS), Fourier transform (FT), short-time Fourier transform (STFT). To conclude we suggest how to validate the instrumentation with comparative experiments and which analysis method to adopt when investigating microfluctuations;in particular we suggest a new statistical approach. [D0I: 10.2971/je0s.2011.11003]

Keywords: accommodation dynamics, wave-front aberrometer, auto refractometer, infrared optometer, microfluctuations, eye

\section{INTRODUCTION}

The capability of the human eye in changing its dioptric power is called accommodation and is a result of a change in shape of the crystalline lens. The accommodation allows the eye to focus at different distances i.e., in normal young eyes, from $0.01 \mathrm{~m}(10 \mathrm{D})$ to infinity $(0 \mathrm{D})$.

While fixating at an immobile object, the accommodation is not perfectly constant. In fact, the accommodation presents small fluctuations, so called microfluctuations, with an amplitude inferior to $1 \mathrm{D}$ and a frequency up to a few $\mathrm{Hz}$. In particular, the microfluctuations with a frequency lower than $1 \mathrm{~Hz}$ are usually defined as the low frequency component (LFC) and fluctuations above or equal $1 \mathrm{~Hz}$ are defined as the high frequency component (HFC) of microfluctuations. Since Collins [1] measured that kind of signals for the first time in 1937, a lot of research has been dedicated to this phenomenon.

Microfluctuations are a difficult phenomenon to explore. This is mainly due to the complexity of the system that generates microfluctuations and to practical difficulties in their measurement.

In order to model the accommodation system it is often necessary to adopt a holistic approach including physiological, neurological and psychological phenomenon involved in vision. Schor [2] introduced a sophisticated model of the ac- commodation. In Schor's model, accommodation is driven by a combination of body and retina referenced stimuli. Body referenced stimuli are mainly involved in coarse processes of accommodation whereas retina referenced stimuli mainly act in the fine-tuning of accommodation setting. A substantial amount of data reported in the literature indicates that complex, non-linear behavior of accommodation could occur in many circumstances [3]-[5]. At the psychological level there are adaptive learning effects, as Stark showed in 1968 [6], since the subjects could anticipate stimulus changes, which was the case when the stimulus varies periodically in time. In this case, there is a reduction of the phase lag and this may enhance the gain as well [7].

It is difficult to compare results of difference experiments when there are several influencing variables and most of them are often not controlled Microfluctuations are in fact affected by: pupil diameter [8]-[10], distance of the target [11] -[18], age of the eye [15], target form and contrast [19]-[24], target luminance $[21,25,26], \mathrm{bi} /$ monocular observation of the target $[9,15,27,28]$, astigmatism of the eye [11]-[13]-[27, 29], visual fatigue [12, 21, 23]-[30]-[32], cardiopulmonary signals [33]-[37]. All these experiments share the same dependent variables, i.e. microfluctuations parameters, focusing on the effect of one or few independent variables and leaving all the others as extraneous variables. Moreover, even in controlled conditions, different patients could have different responses 
characteristic of the persons; this introduce another extraneous variables. For these reasons only a statistic approach can be used to compare the results on two samples: in two enough big and random samples of a population, the extraneous variables have the same effect on the average of the dependent variables calculated on the two populations. Finally, a further difficulty in comparing different results is due to methods used in the experiments. In particular, different instruments and methods of analysis are used to estimate the dependent variables.

A basic open question concerns the sources of microfluctuations. The recorded fluctuating signal could be due to several causes: crystalline lens accommodation, movement of the eye (retina, crystalline, eyeball shape, cornea) due to body movements (e.g. heartbeat, eye movements (e.g. microsaccades), breathing) and artifacts caused by the measuring instrument. For instance, Heijde et al. [38] used a continuous ultrasonographic biometry to measure changes in the morphology of the eye during steady state accommodation. Results show that fluctuations with a LFC were present in anterior chamber depth, lens thickness and vitreous length but not in the axial length, proving that only the lens was involved. In contrast with the findings of investigators using optical methods, no HFC was present in fluctuations of the anterior chamber depth and lens thickness. However, a small HFC was found in recordings of the vitreous and axial length, which appeared to correspond with the heart rate. Heijde et al. conclude that probably IR optometers are more sensitive to artifacts, e.g. caused by head and eye movements, than is the case in their ultrasonographic system. The debate concerning the existence of a peak in microfluctuations at about $2 \mathrm{~Hz}$ (HFC) is still spread within researches where IR optometers have been used. On one side Collins 1937, Campbell 1959, Kotulak 1986, Toshida 1998 and Iskander 2004 [1, 8, 16, 39,35 ] have reported a peak in HFC whereas on the other side in Heron 1972, Denieul 1978, Denieul 1980, Denieul 1982a and Mira-Agudelo 2009 [14, 20, 21, 28, 40] the HFC peak is absent in the signal of microfluctuations. Other researchers as Miege 1988, Schultz 2009 or Muma 2010 [17, 41, 37] occasionally report a HFC peak in records of microfluctuations. HFC can thus be an artifact produced by the measurement instrument, e.g. by mechanical part resonating or actively moving in the HFC bandwidth. HFC can be an artifact produced by the heartbeat, breathing or other rhythmical physiological systems that have harmonics in the HFC bandwidth: the instrument used should be insensitive to other values than the accommodation. For example, breathing at $1.5 \mathrm{~Hz}$ brings to a periodical movement of the eye at that frequency, and, if the instrument is sensitive to displacements of the eye, a false HFC microfluctuating accommodation signal is recorded. Similarly, if a person, like the patient, is in physical contact with the instrument, it could stimulate on the device vibrations produced by her/his rhythmical physiological movements. Moreover, HFC cannot be seen if the sampling rate of the instrument and the accuracy are not sufficient.

Charman and Heron [42] show how the results of Bour [19] are perhaps surprising if compared with the results of Tucker et al. [43]. While the former concludes that fluctuations do not seem to be systematically dependent on either the grating frequency or contrast of the visual target, the later states that sensitivity to defocus is undoubtedly affected by both these parameters.

Studies on microfluctuations as a function of age, e.g. [44, 45], have also reported decreases in fluctuations with increasing age; however, the increase in fluctuations in the fourth decade of life found by Anderson et al. [46] are not reported in Heron 1995 and Mordi 2004.

Zhu et al. [36] conclude that higher order components of the eye wave-front exhibit fluctuations similar in frequency to those of defocus; conversely, Hofer et al. [47] state that the shape of the spectrum for higher-order aberrations is generally different from for the one of microfluctuations.

Numerous investigations have shown evidence of a correlation between the HFC of accommodation fluctuations and the pulse rate [33, 34, 48, 49]; controversially Hampson et al.'s findings [50] do not support the results of these studies.

Though Hofer et al. [47] have excluded a number of potential causes for the fluctuations in the wave-front aberration, such as instrument artifact, random noise, and direct optical effects of eye rotation and translation; they conclude that their experiments to date have not identified the origin of the fluctuations in the eye with certainty.

In our opinion, much of the conflicting results are due to the different and sometimes inappropriate methods used to record and analyze microfluctuations. In this work, we review the measurement instruments and the signal analysis methods applied in research on microfluctuations. We finally suggest ways to improve the methodology of recording and analyzing microfluctuations.

\section{MEASUREMENT INSTRUMENTS}

In our review of the literature, we could barely find the usage of the same measurement instrument in different studies. The market, in fact, offers several instruments to record accommodation and some of them measure microfluctuations too, e.g. Speedy-K ver. MF-1 produced by Righton. In the other cases, either standard instruments have been modified for the specific purpose or laboratories developed their own apparatus.

In early times subjective methods have been developed. Among others, the cross-cylinder and the spatially resolved refractometer (SSR) are the most documented techniques [51] -[55]. Subjective methods have the disadvantage that the measurement performance depends on the patient's ability to precisely complete the task. Moreover, often the measurement process is very time consuming (up 15 minutes), which 
makes such methods inappropriate for use in a clinical environment [55].

On the other hand, objective methods, usually allow highspeed measurement rate, high accuracy and repeatability. Given the obvious advantages of objective methods, objective methods almost completely replaced the subjective ones.

In addition to the previous classification between subjective and objective methods, we individualized three families of instruments used to analyze microfluctuations: wave-front aberrometer, classical IR autorefractometer, and ultrasound.

Wave-front recording accommodation by means of wavefront aberrometers enables to compute high-order aberrations, usually defined mathematically by a series of polynomials, that are, the Zernike polynomials [56]. Therefore, additional information beyond spherical defocus and astigmatism $[57,58]$ is available on the optics of the eye when using wave-front aberrometer. Thanks to wave-front aberrometer it is thus possible to analyze the microfluctuations of each of the Zernike polynomials and eventually combine them together to have a total defocus error. On the other side, current wave-front aberrometer technologies, e.g. the ShackHartmann method [59] and the laser ray-tracing sensors [60, 61], suffer from some inaccuracies that may limit a wider expansion in the clinical environment [55]. Several innovative approaches are ripening to overcome the limits of standard wave-front sensing techniques. Curvature sensing [62, 63], pyramid sensing [64]-[66] and interferometry (e.g. MachZehnder interferometer [67]-[69], shearing and multiple shearing interferometry [70]-[76]) these currently represent the most reliable methods to revise and improve the measurement and reconstruction of the wave-front aberrations of human eye [55]. This family is therefore including all those device that allow to measure the weve-front aberration of the eye, i.e. the aberration in every point of the whole aperture of the eye.

In the classical IR autorefractometer family, we catalog all the other optical methods that are not in the wave-front aberrometer family. In this family, we find autorefractometers that enable continuous sampling of accommodation. Output of autorefractometers is limited to spherical defocus and astigmatism and do not offer the possibility to track Zernike polynomials of an order higher than two. An advantage of this family, compared to the wave-front aberrometers, is a lower level of technology of the instrument: e.g., autorefractometers do now use Hartmann masks or microlens arrays. Some examples of devices belonging to this family are described in the papers of Monticone 2010b, Monticone 2010c, Arnulf 1981, Kruger 1979, Krueger 1973 and Suryakumar 2007 [13]-[77]-[81].

In the ultrasound family, we catalog instruments that allow tracking the microfluctuations using an ultrasonographic method. The main advantage of ultrasonography, for our purpose, is the completely different approach compared to the other optical methods. An additional advantage is that ultrasonography provides a means for examining eyes that cannot be optically inspected because of corneal opacities, cataracts, hemorrhages and the like [82]. Some examples of devices belonging to this family are described in the following papers: [38]-[82]-[85].

\section{ANALYSIS METHODS}

As we discussed, microfluctuations are fluctuating over time and their spectrum (frequency components) is usually divided in LFC and HFC. Most of the studies about microfluctuations ignore that the spectrum of microfluctuations could change over time. However, some studies that analyze the variations of the spectrum conclude that it is not stationary $[35,37,50,86]$. The methods used to analyze microfluctuations should thus test for the stationary of the spectrum and then analyze the microfluctuations compatibly with the result.

Reviewing the literature, we individualized three main families of methods used to analyze microfluctuations: root mean square (RMS), Fourier transform (FT), short-time Fourier transform (STFT).

The RMS value is equivalent to the standard deviation of the signal, if the average value that biases the signal is taken as zero. The main advantage of these methods is that there is no need of following the signal as defined by Nyquist. The accuracy of the result is just due to the number of samples measured on the signal, thus it is enough to have a long signal compared to the sampling rate. However, the disadvantage is that it is appropriate to use these methods only when the spectrum of the signal is stationary. The result of this method is in fact just one number; thus it hides the evolution of the spectrum over time. In every case, even under the stationary hypothesis, the RMS value does not give any information about the shape of the spectrum of the signal, e.g., about the existence of HFC. Some examples of studies that used this method are found in Arnulf 1960, Denieul 1978, Denieul 1980, Denieul 1982a, Krueger 1978, Kotulak 1986, Bour 1981, Schultz 2009, Anderson 2010, Winn 1992 and Candi 2007 [11, $14,15,16,19,20,21,41,46,48,87]$. Figure 2 , in the graph on the top, represents a normalized real signal of microfluctuations of the accommodation. In this case the RMS value would roughly be 0.5 , meaning that the average fluctuation of the signal is $50 \%$ of the normalization value.

The FT (in this family we include all the methods that results in a spectrum of the signal) can be used only if the Nyquist's theorem is satisfied. In order to avoid aliasing problems, the sampling rate has to be at least the double of the maximum frequency contained in the signal. Moreover, it is appropriate to use the FT only under the hypothesis of a stationary spectrum. In case of the spectrum varying in time, the FT computes an average of spectra of the signal over time. Averaging the variation of the spectrum over time leads to an underestimation of the peaks: e.g. if a peak is stationary for half of the length of the signal and then disappears, the corre- 


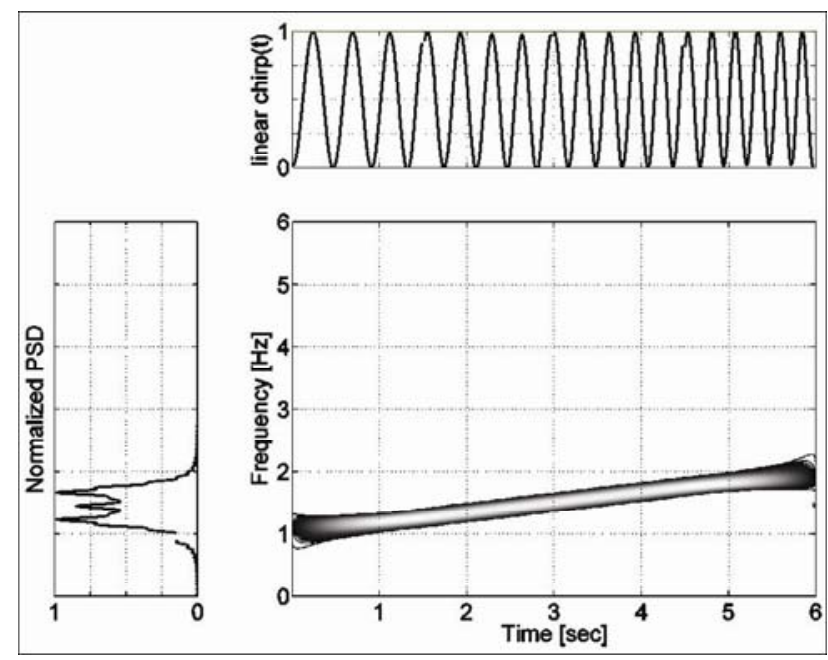

Fig. 1 In the top figure, a linear chirp function is plotted as function of time. Since the frequency of the sinusoidal oscillation is linearly increasing with time, the spectrogram plot is a straight line with a positive slope. The power spectrum density (PSD) of the whole signal (the chirp function) is plotted in the left graph. One can state that no dominant peak is present in the PSD plot. In fact we should theoretically have a flat band due to the shift of the peak. The standard FFT analysis would thus hide the presence of a peak due to its non-stationary behavior.

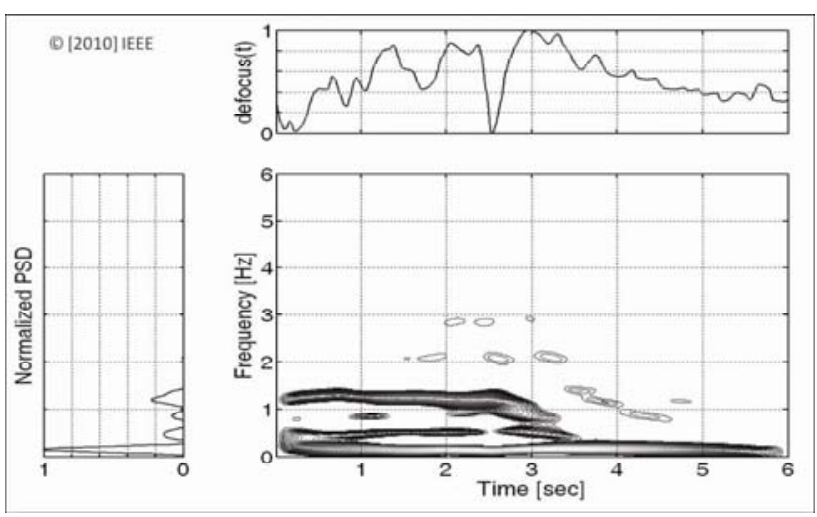

Fig. 2 (๔ [2010] IEEE [37]). This figure shows a microfluctuations signal on the top and his PSD and spectrum calculated in the same way as for the chirp function in figure 1. As it is possible to see the microfluctuations signal is not stationary, especially the HFC. For this reason, for instance, the peak at about $1.2 \mathrm{~Hz}$ is roughly underestimated by a factor two in the PSD chart.

sponding peak on the FT spectrum will measure half of the original amplitude (e.g. in Figure 2). This can be an explanation for the diatribe about the existence of the HFC. It could sometimes even be impossible to detect the peak: e.g. if the peak changes its frequency over time (e.g. in Figure 1), the FT spectrum will have a constant value over the entire band brushed by the peak. Some examples of studies that used this family of method are reported in Schultz 2009, Iskander 2004, Hofer 2001, Heijde 1996, Zhu 2004, Mira-Agudelo 2009, Candi 2007 and Hampson 2005 [28, 35, 36, 38, 41, 47, 50, 87].

In a middle state between the RMS and the FT methods are some studies where the RMS value is calculated after filter- ing the signal in two (i.e., LFC and HFC) or three bands. In this case, the results have low frequency accuracy but a better accuracy of the RMS values calculated on each bandwidth compared to each single FT spectral lines. Some examples of studies that used this method are Gray 2000, Toshida 1998 and Mira-Agudelo 2009 [28, 39, 88].

The STFT (in this family we include all the methods that bring to a spectrogram, i.e., evolution of spectrum over time (e.g. Figure 1) requires that the sampling rate satisfies the Nyquist's theorem. However, the signal is not required to be stationary. STFT consist in several FT using windows of the signal. In this way the spectrum has to be hypothesized stationary only for the short time of the length of the window (precisely, the Nyquist's theorem has to be satisfied even on the signal of the spectrum over time, e.g. if the spectrum is changing at $0.5 \mathrm{~Hz}$ we should have a temporal resolution of at least $1 \mathrm{~s}$ ). The length of the windows has to be chosen carefully because it determines a tradeoff between resolution in time and resolution in frequency. Some examples of studies that used methods of this family are Iskander 2001, Iskander 2004 and Muma 2010 [35, 37, 86].

Theoretically, the same approach of analyzing the signal multiplied by a moving window can be applied to the RMS methods (RMS / FT = moving RMS / STFT). In this case, the results would be the RMS values as function of time. The trade off to face now is between the temporal accuracy and the precision of the value even if the first is linear while the second goes as the square root of the length of the window expressed in number of samples. This value has not been used since it requires about the same recording speed as to compute a STFT but is gives much less information about microfluctuations.

\section{CONCLUSIONS}

We discussed the methods used for recording and analyzing microfluctuations. Contrasting results of different studies could arise from the use of different methods. We now give some conclusion to try to improve these methods: how to better validate the measurement instruments and which analysis method to adopt. Finally, we suggest the concepts behind a new analysis method that, in our opinion, should be, at the state of the art, the most appropriate to deal with microfluctuations.

Concerning the recording devices, the literature offers in part descriptions of the devices; however, we were not able to find any empirical study comparing instruments, especially comparing instruments belonging to different families. A transversal study on different instruments and families is necessary in order to validate conclusion like the one of Heijde et al. [38], stating that IR optometers are probably more sensitive to artifacts, than ultrasonographic systems. For instance, it would be easy, perhaps thanks to a beam splitter, to assess the same measurements contemporaneously with both a classical IR autorefractometer and a wave- 


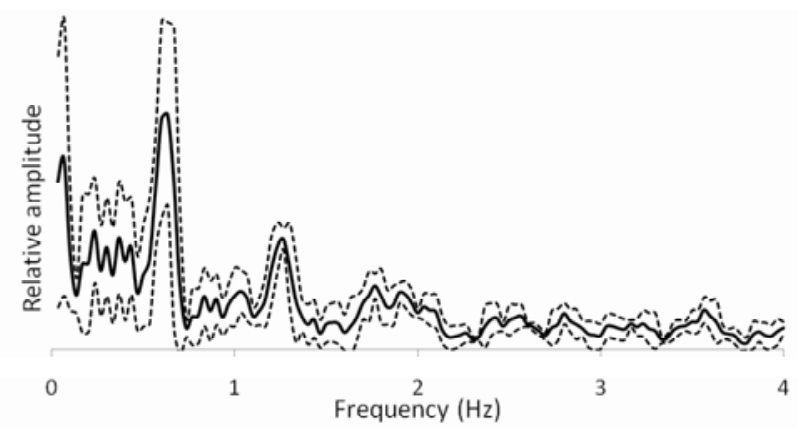

Fig. 3 In this figure is given an illustrative example of microfluctuations spectrum (continuous line) and its statistical dispersion (the dotted lines indicate the confidential intervals).

front aberrometer. Possibly this comparison analysis should be done on a variety of instruments including an ultrasonographic system. Due to mechanical constrains, the latter system is probably the most difficult to use at the same time with an optical instrument. However, it is still possible to limit the variation of microfluctuations due to noninstrumental factors, measuring consecutively the subject, kept in steady conditions, with different instruments. Of course, even the data analysis method of the results given by the different instruments has to be the same and has to be opportunely chosen as discussed before. Using such a designed experiment setup, the results have to be qualitatively and quantitatively compatible; in particular, in the case of contemporary measurement with different instruments, the results have to be the same. Possible differences would be attributed to inaccuracies and artifacts of the recording instruments.

Concerning the methods of data analysis, we showed how the STFT family is the most appropriate since the method considers the possibility of a non-stationary spectrum of microfluctuations. However, we think that the STFT methods, as much as the FT too, lack a complete statistical approach. In statistics, it is quite meaningless to compare average values without knowing their variances. For the sake of simplicity, but without losing the generalization to STFT methods, let us focus on the spectrum resulted by a FT analysis of a microfluctuation signal. In such a graph, the presence of a peak does not mean that it corresponds with a real signal: it could be just a case. To statistically judge if the peak is a signal, we should have the information of its variance and then run a power analysis. Postponing the mathematical formalization to a further study, the variance of the spectrum should be depicted on the spectrum graph, e.g. plotting two lines of plus/minus one standard deviation (Figure 3). In the spectrogram, we just add the temporal dimension, thus, instead of tree lines, we have tree surfaces. Only comparing the peak amplitude with the variance we could state that such signal exists with a certain, possibly high, probability. Without this statistical approach one researcher could for instance misinterpret HFC as noise or vice versa.

\section{ACKNOWLEDGEMENTS}

This study was supported by grants from the Swiss National Science Foundation (SNSF) No 320030-116423.

The authors would like to acknowledge Muma M, Iskander DR and Collins MJ for allowing the reproduction of their results (Figure 2) and in particular Muma $\mathrm{M}$ for providing us Figure 1.

\section{References}

[1] G. Collins, "The electronic refractometer" Brit. J. Physiol. Opt. 1, 30-40 (1937).

[2] C.M. Schor, L.K. Cormack, S.B. Stevenson, "Negative feedback control model of proximal convergence and accommodation" Ophthal. Physl. Opt. 12 307-318 (1992).

[3] F.W. Campbell, "The accommodation response of the human eye" Brit. J. Physiol. Opt. 16, 188-203 (1960).

[4] W.D. O'Neill, C.K. Sanathanan, and J.S. Brodkey "A minimum variance, time optimal, control system mode of human lens accommodation" IEEE Transactions on Systems Science and Cybernetics 5, 290-299 (1969).

[5] D. Shirachi,J. Liu, M. Lee "Accommodation dynamics. 1. Range nonlinearity" Am. J. Optom. Phys. Opt. 55, 631-641 (1978).

[6] L. Stark, Neurological control systems: studies in bioengineering (Plenum Press, New York, 1968).

[7] 0. Franzen, H. Richter, and L. Stark, "Accommodation and vergence mechanisms in the visual systems" in Proceedings of the First International Symposium on Accommodation/Vergence Mechanisms in the Visual System, 129-140 (Stockholm, 2000).

[8] F.W. Campbell, J.G. Robson, and G. Westheimer, "Fluctuations of accommodation under steady viewing conditions" J. PhysiolLondon 145, 579-594 (1959).

[9] F.W. Campbell, and G. Westheimer, "Dynamics of the accommodation responses of the human eye" J. Physiol-London 151, 285295 (1960).

[10] W.N. Charman, and H. Radhakrishnan, "Accommodation, pupil diameter and myopia" Ophthal. Physl. Opt. 29, 72-79 (2009).

[11] A. Arnulf, and 0. Depuy, "Contribution a l'étude des microfluctuations d'accommodation de l'œil", Rev. Opt-Paris 39, 195-208 (1960).

[12] A. Arnulf, J. Santamaría, and J. Bescós, "El sistema optico del ojo primerescalon de la sensacion visual" El optico profesional 191, 13-20 (1977).

[13] A. Arnulf, J. Santamaría, and J. Bescós, "A cinematograph method for the dynamic study of the image formation by the human eye. Microfluctuations of the accommodation" J. Opt-Paris 12, 123-128 (1981).

[14] P. Denieul, "Effects of stimulus vergence on mean accommodation response, microfluctuations of accommodation and optical quality of the human eye", Vision Res. 22 561-569 (1982).

[15] H. Krueger 'Schwankungen der Akkommodation des menschlichen auges bei mon. und binokular Beobachtung" Albrecht v. Graefes Arch. Klin. Exp. Ophthal. 205, 129-133 (1978).

[16] J.C. Kotulak, and C.M. Schor "Temporal variations in accommodation during steady-state conditions" J. Opt. Soc. Am. 3, 223-227 
(1986).

[17] C. Miege, and P. Denieul "Mean response and oscillations of accommodation for various stimulus vergences and feedback control of the accommodative system" Ophthal. Physl. Opt. 8, 165-171 (1988).

[18] S. Usui, L. Stark "Sensory and motor mechanism interact to control amplitude of pupil noise" Vision Res. 18, 505-507 (1978).

[19] L.J. Bour, "The influence of the spatial distribution of a target on the dynamic response and fluctuations of the accommodation of the human eye" Vision Res. 21, 1287-1296 (1981).

[20] P. Denieul, "Dynamic study of microfluctuations of accommodation with a high sensitive infrared optometer" in Optica Hoy y Manana, Proceedings of the ICO-11 Conference, 51-54 (ICO, Madrid, 1978).

[21] P. Denieul, Etude des fluctuations d'accommodation de l'oeil par Optométrie Infra Rouge (Thesis, L'Université de Paris-Sud, 1980).

[22] P. Denieul, "Influence de la dimension des tests sur l'accommodation" L'0ptométrie 28, 12-13 (1982).

[23] P. Denieul, and F. Corno, "Accommodation et contraste" L'Optométrie 32, 4-8 (1986).

[24] S. Phillips, L. Stark, "Blur: a sufficient accommodative stimulus" Doc. Ophthalmol. 43, 65-89 (1977).

[25] M. Alpern, "Variability of accommodation during steady fixation at various levels of illuminance" J. Opt. Soc. Am. 48, 193-197 (1958).

[26] C.M. Schor, C.A. Johnson, R.B. Post, "Adaptation of tonic accommodation" Ophthal. Physl. Opt. 4, 133-137 (1984).

[27] J. Santamaría, A. Plaza, J. Bescós, "Dynamic recording of the binocular point spread function of the eye optical system" 0pt. Appl. 14, 341-347 (1984).

[28] A. Mira-Agudelo, L. Lundstroem, and P. Artal, "Temporal dynamics of ocular aberrations monocular vs binocular vision" Ophthal. Physl. Opt. 29, 256-263 (2009).

[29] L.R. Stark, N.C. Strang, D.A. Atchison, "Dynamic accommodation response in the presence of astigmatism" J. Opt. Soc. Am. A. 20, 2228-2236 (2003).

[30] A. Arnulf, 0. Depuy, and F. Flamant, "Les microfluctuations d'accommodation de l'oeil et l'acuité visuelle pour les diamètres pupillaires naturals" C. R. Hebd seanc Acad Sci Paris 232, 349-350 (1951).

[31] T. Iwasaki, and S. Kurimoto, "Objective evaluation of eye strain using measurements of accommodative oscillation" Ergonomics $30,581-587$ (1987).

[32] P.P. Monticone, and M. Menozzi, Eye stress, flickering and microfluctuations (GfA Spring Congress, Darmstadt, 24-26 March 2010).

[33] B. Winn, J.R. Pugh, B. Gilmartin, H. Owens, "Arterial pulse modulates steady-state ocular accommodation" Curr. Eye Res. 9, 971975 (1990).

[34] M.J. Collins, B. Davis, and J. Wood, "Microfluctuations of steadystate accommodation and the cardiopulmonary system" Vision Res. 35, 2491-2502 (1995).

[35] D.R. Iskander, M.J. Collins, M.R. Morelande, and M. Zhu, "Analyzing the dynamic wave-front aberrations in the human eye" IEEE Transactions on Biomedical Engineering 51, 1969-1980 (2004).
[36] M. Zhu, M.J. Collins, D.R. Iskander, "Microfluctuations of wavefront aberrations of the eye" Ophthal. Physl. Opt. 24, 562-571 (2004).

[37] M. Muma, D.R. Iskander, and M.J. Collins, "The Role of Cardiopulmonary Signals in the Dynamics of the Eye's Wave-front Aberrations" IEEE Transactions On Biomedical Engineering 57, 373-383 (2010).

[38] G.L. van der Heijde, A.P.A. Beers, and M. Dubbleman, "Microfluctuations of steady state accommodation measured with ultrasonography" Ophthal. Physl. Opt. 16, 216-221 (1996).

[39] K. Toshida, F. Okuyama, T. Tokoro, "Influences of the Accommodative Stimulus and Aging on the Accommodative Microfluctuation" Optometry Vision Sci. 75, 221-226 (1998).

[40] G. Heron, A study of accommodation using an infra-red optometer (MSC Thesis, University of Manchester, 1972).

[41] K.E. Schultz, Accommodative microfluctuations, crystalline lens tension, ciliary body thickness, and refractive error in children (MSc. Thesis, Graduate School of The Ohio State University 2009).

[42] W.N. Charman, "Fluctuation in accommodation: a review" Ophthal. Physl. Opt. 9, 153-164 (1988).

[43] J. Tucker, W.N. Charman, P.A. Ward, "Modulation dependence of the accommodation response to sinusoidal gratings" Vision Res. 26, 1693-1707 (1986).

[44] G. Heron, and C. Schor, "The fluctuations of accommodation and ageing" Ophthal. Physl. Opt. 15, 445-449 (1995).

[45] J.A. Mordi, and K.J. Ciuffreda, "Dynamic aspects of accommodation: age and presbyopia" Vision Res. 44, 591- 601 (2004).

[46] H.A. Anderson, A. Glasser, R.E. Manny, and K.K. Stuebing, "Agerelated changes in accommodative dynamics from preschool to adulthood" Invest. Ophth. Vis. Sci. 51, 614-622 (2010).

[47] H. Hofer, P. Artal, B. Singer, J.L. Aragon, and D.R. Williams, "Dynamics of the eye's wave aberration" J. Opt. Soc. Am. A. 18, 497-506 (2001).

[48] B. Winn, B. Gilmartin "Current perspective on microfluctuations of accommodation", Ophthal. Physl. Opt. 12 252-256 (1992).

[49] A.S. Eadie, J.R. Pugh, and B. Winn, "The use of coherence functions in the study of ocular mechanisms" Ophthal. Physl. 0pt. $15,311-317$ (1995).

[50] K.M. Hampson, I. Munro, C. Paterson, and C. Dainty, "Weak correlation between the aberration dynamics of the human eye and the cardiopulmonary system" J. Opt. Soc. Am. A. 22, 1241-1250 (2005).

[51] J.C. He, S. Marcos, R.H. Webb, and S.A. Burns, "Measurement of the wave-front aberration of the eye by a fast psychophysical procedure" J. Opt. Soc. Am. A. 15, 2449-2456 (1998).

[52] H.C. Howland, "The history and methods of ophthalmic wavefront sensing"।. Refract. Surg. 16, 552-553 (2000).

[53] R.I. Calver, M.J. Cox, and D.B. Elliot, "Effect of aging on the monochromatic aberrations of the human eye" J. Opt. Soc. Am. A. 16, 2069- 2078 (1999).

[54] S.A. Burns, "The spatially resolved refractometer" J. Refract. Surg. 16, 566-569 (2000)

[55] M. Lombardo, and G. Lombardo, "New methods and techniques for sensing the wave aberrations of human eyes" Clin. Exp. Optom. 92, 176-186 (2009). 
[56] ISO 24157:2008 "Ophthalmic optics and instruments: Reporting aberrations of the human eye", International Standards Organization (2008).

[57] D.A. Atchison, "Recent advances in measurement of monochromatic xaberrations of human eyes" Clin. Exp. 0ptom. 88, 5-27 (2005).

[58] L.N. Thibos, X. Hong, A. Bradley, X. Cheng, "Statistical variation of aberration structure and image quality in a normal population of healthy eyes"' J. Opt. Soc. Am. A. 19, 2329-2348 (2002).

[59] R. Platt, and R. Shack, "History and principles of Shack-Hartmann wave-front sensing" J. Refract. Surg. 17, 573-577 (2001).

[60] R. Navarro, and M.A. Losada, "Aberrations and relatively efficiency of light pencils in the living human eye" Optom. Vis. Sci. 74, 540-547 (1997).

[61] R. Navarro, E. Moreno-Barriuso, "Laser ray-tracing method for optical testing" Opt. Lett. 24, 1-3 (1999).

[62] F. Roddier, "Curvature sensing and compensation: a new concept in adaptive optics" Appl. Optics. 27, 1223-1225 (1988).

[63] F. Diaz-Douton, J. Pujol, M. Arjona, and S.0. Luque, "Curvature sensor for ocular wave-front measurement" Opt. Lett. 31, 22452247 (2006).

[64] R. Ragazzoni, J. Farinato, "Sensitivity of a pyramidic wave front sensor in closed loop adaptive optics" Astron. Astrophys. 350, L23-L26 (1999).

[65] I. Iglesias, R. Ragazzoni, Y. Julien, and P. Artal, "Extended source pyramid wave-front sensor for the human eye" Opt. Express. 10, 419-428 (2002).

[66] S.R. Chamot, C. Dainty, and S. Esposito, "Adaptive optics for ophthalmic applications using a pyramid wave-front sensor" 0 pt. Express. 2, 518-526 (2006).

[67] T. Shirai, T.H. Barnes, T.G. Haskell, "Adaptive wave-front correction by means of alloptical feedback interferometry" Opt. Lett. 25, $773-775$ (2000).

[68] T. Shirai, "Liquid-crystal adaptive optics based on feedback interferometry for highresolution retinal imaging" Appl. Optics 41, 4013-4023 (2002).

[69] T. Shirai, T.H. Barnes, "Adaptive restoration of a partially coherent blurred image using an all-optical feedback interferometer with a liquid-crystal device" J. Opt. Soc. Am. A. 19, 369-377 (2002).

[70] H.H. Lee, J.H. You, and S.H. Park, "Pak Phase-shifting lateral shearing interferometer with two pairs of wedge plates" 0 pt. Lett. 28, 2243-2245 (2003).

[71] S. Velghe, J. Primot, N. Guerineau, M. Cohen, B. Wattelier, "Wavefront reconstruction from multidirectional phase derivatives generated by multilateral shearing interferometers" Opt. Lett. 30, 245 -247 (2005).

[72] C. Siegel, F. Loewenthal, J.E. Balmer, "A wave-front sensor based on the fractional Talbot effect" Opt. Commun. 194, 265- 275 (2001).
[73] R. Sekine , T. Shibuya, K. Ukai, S. Komatsu, M. Hattori, T. Mihashi, N. Nakazawa et al., "Measurement of wave-front aberration of human eye using Talbot image of twodimensional grating" Opt. Rev. 13, 207- 211 (2006).

[74] L. Warden, Y. Liu, P.S. Binder, A.W. Dreher, L. Sverdrup "Performance of a new binocular wave-front aberrometer based on a selfimaging diffractive sensor" J. Refract. Surg. 24, 188-196 (2008).

[75] L.S. Horwitz, "System and method for wave-front measurement" US Patent 6,781,681 (2004).

[76] L.S. Horwitz, "Ocular biometer" US Patent 5,963,300 (1999).

[77] P.P. Monticone, and M. Menozzi, "Instrument for high-speed recording of accommodation of the human eye" Biomed. Tech. 55, 83-88 (2010).

[78] P.P. Monticone, M. Menozzi, and S. Martin, High speed and high accuracy accommodation tracker (CfA Spring Congress, Darmstadt, 24-26 March 2010).

[79] P.B. Kruger, "Infrared recording retinoscope for monitoring accommodation" Am. Optom. Phys. Opt. 56, 116-123 (1979).

[80] H. Krueger, "An apparatus for continuous, objective measurement of refraction of the human eye" Opt. Acta 20, 277-285 (1973).

[81] R. Suryakumar, J.P. Meyers, E.L. Irving, W.R. Bobier, "Application of video-based technology for the simultaneous measurement of accommodation and vergence" Vision Res. 47, 260-268 (2007).

[82] J.W. Kohl, "Ultrasonography in ophthalmology" Western J. Med. 120, 234-234 (1974).

[83] F.R. de Vries, G.L. van der Heijde, H.G. Goovaerts, "System for continuous high-resolution measurement of distances in the eye" J. Biomed. Eng. 9, 32-37 (1987).

[84] A.P.A. Beers, and G.L. van der Heijde, "In vivo Determination of the Biomechanical Properties of the Component Elements of the Accommodation Mechanism" Vision Res. 34, 2897-2905 (1994).

[85] A.P.A. Beers, and G.L. van der Heijde, "Analysis of accommodation function with ultrasonography" Doc. Ophthalmol. 92, 1-lo (1996).

[86] D.R. Iskander, M.R. Morelande, and M.J. Collins, "Estimating the dynamics of aberration components in the human eye" in Proceedings of the 11th IEEE Workshop Statistical Signal Processing (SSP 2001), 241-244 (IEEE, Singapore, 2001).

[87] T.R. Candi, and S.R. Bharadwaj, "The stability of steady state accommodation in human infants" J. Vision 4, 1-16 (2007).

[88] L.S. Gray, B. Gilmartin, and B. Winn, "Accommodation microfluctuations and pupil size during sustained viewing of visual display terminals" Ophthal. Physl. Opt. 20, 5-10 (2000). 